\title{
EFFECTS OF SNPS IN THE COL1A1 AND METHYLENETETRAHYDROFOLATE REDUCTASE GENES ON BMD IN POSTMENOPAUSAL WOMEN IN MALTA
}

\author{
Vidal $\mathrm{C}^{1}$, Brincat $\mathrm{M}^{2}$, Xuereb-Anastasi $\mathrm{A}^{1,3^{*}}$
}

\begin{abstract}
*Corresponding Author: Professor Angela Xuereb-Anastasi, Ph.D., DNA Laboratory, Department of Pathology, University of Malta Medical School, G’Mangia, MSD06, Malta; Tel.: +35625551882; Fax:+356-21235638; E-mail: angela.a.xuereb@um.edu.mt
\end{abstract}

\section{ABSTRACT}

Two common single nucleotide polymorphisms (SNPs) within the COL1A1 gene and the C677T variant within the methylenetetrahydrofolate reductase (MTHFR) gene have been studied for correlation with bone mineral density (BMD) in 126 postmenopausal Maltesewomen (55.6 \pm 7.1 years). All polymorphisms were analyzed by polymerase chain reaction-restriction fragment length polymorphism (PCR-RFLP), while BMD at the lumbar spine (LS), femoral neck (FN), Ward's triangle and trochanter was measured by dual energy X-ray absorptiometry (DEXA).

The observed genotype frequencies were similar to those in other populations and were in HardyWeinberg equilibrium. No association was observed between polymorphisms in the COL1A1 gene and BMD, even after adjustment for age, body mass index (BMI) and years since menopause. The $\mathrm{C}$ allele of the C677T variant of the MTHFR gene had a negative effect on trochanter BMD when testing for genetic models of dominant and recessive alleles (independent sample $t$-test: $p=0.03$ ). Genotype frequencies of both genes did not differ significantly between normal women and those with a low BMD at either the LS or FN.

\footnotetext{
${ }^{1}$ Department of Pathology, University of Malta Medical School, G'Mangia, Malta

2 Department of Obstetrics and Gynaecology, University of Malta Medical School, G'Mangia, Malta

${ }^{3}$ Institute of Health Care, University of Malta, G’Mangia, Malta
}

Keywords: Bone mineral density (BMD); Bone loss; COL1A1; Maltese population; Methylenetetrahydrofolate reductase (MTHFR); Osteoporosis

\section{INTRODUCTION}

The search for genes responsible for most complex diseases is a challenging task in human genetics since these diseases result from complex interactions between the environment and susceptibility alleles in multiple genes. Several single nucleotide polymorphism (SNPs) within candidate genes have been studied for association with increased susceptibility to osteoporosis and/or fracture risk $[1,2]$. These candidate genes are primarily involved in biological processes of bone physiology and include genes for various receptors [3-6], cytokines [7], growth factors [8-10] and structural proteins [11]. Genes not directly involved in bone biology have also been studied $[12,13]$.

Type I collagen is the most abundant and ubiquitously expressed of the collagen superfamily of proteins that are the most abundant proteins in the human body. Genes that encode the $\alpha 1$ (I) and $\alpha 2(\mathrm{I})$ chains are located on chromosomes 17 and 7, respectively, and several polymorphisms and mutations have been identified and associated with diseases such as osteogenesis imperfecta and osteoporosis [14,15]. In 1996, a novel polymorphism in the $\mathrm{Sp} 1$ binding site of the COL1A1 gene promoter was associated with low bone mass and increased risk of vertebral fracture [11]. Similar studies in other populations produced conflicting results [16- 
21]. Two new polymorphisms in the regulatory region of the COL1A1 gene, to which primary osteoblast nuclear proteins bind, were recently identified, of which a $\mathrm{G} \rightarrow \mathrm{T}$ transversion at position -1997 was associated with decreased lumbar bone mineral density (BMD) and to a lesser degree, with femoral BMD in postmenopausal women [22].

Collagen undergoes several posttranslational modifications which include cross-linking and hydroxylation of proline and lysine residues, catalyzed by various isomerases and hydroxylases [15]. High serum homocysteine concentrations may interfere with cross-linking of collagen and thus affect bone quality [23]. Such increased concentrations have been observed in individuals with a low folate status who are homozygous for the C677T variant of the methylenetetrahydrofolate reductase (MTHFR) gene [24] in which substitution from alanine to valine produces a thermolabile enzyme [25]. This common variant has been associated with coronary artery disease [25], neural tube defects [26] and low bone BMD [12,27]. Suggestive linkage of femoral BMD has been reported to locus $1 \mathrm{p} 36$ [28-30], where the MTHFR and other candidate genes for osteoporosis are located [13]. We have studied the Sp1 and $-1997 \mathrm{G} \rightarrow \mathrm{T}$ polymorphisms within the promoter region of the COL1A1 gene and the MTHFR C677T variant for association with BMD in a group of postmenopausal women in Malta.

\section{MATERIALS AND METHODS}

Patient Recruitment. One hundred and twentysix postmenopausal women were recruited from new subjects referred to the Bone Density Unit, Department of Obstetrics and Gynaecology, St. Luke's Hospital, G'Mangia, Malta, for an osteoporosis risk evaluation. All were healthy Caucasians between the ages of 40-75 years [mean \pm standard deviation (SD): $55.6 \pm 7.1$ years]. Informed consent was obtained from all participants and the study was approved by the Research Ethics Committee of the Faculty of Medicine and Surgery, University of Malta, Msida, Malta. All participants answered a detailed questionnaire concerning medical conditions and the use of medications, family history of osteoporosis and dietary/ lifestyle habits, and only those with no medical conditions and not on treatments known to affect bone metabolism, were included. Blood for DNA analysis and biochemical markers of bone turnover was collected from each participant.

According to World Health Organization (WHO) criteria [31] for both lumbar and femoral BMD, 30 (24.4\%) of the participants were osteoporotic at the lumbar spine (LS) $(t$-score $<-2.5), 41(33.3 \%)$ were osteopenic ( $t$-score $<-1.0$ to -2.5$)$ and $52(42.2 \%)$ were normal. At the femoral neck (FN), four $(3.2 \%)$ were osteoporotic, $45(36.3 \%)$ osteopenic and 75 $(60.5 \%)$ were normal. The sample was divided into two groups, one of normal individuals (41.1\%) having both lumbar and femoral $t$-score $>-1.0$ and another of individuals who were osteopenic and/or osteoporotic at one or both anatomical sites (58.9\%). The MTHFR C677T polymorphism was also analyzed in a random sample of 254 cord blood DNA obtained from neonates to determine the frequency of this polymorphism in the Maltese population at birth.

Genetic Analysis. Genomic DNA was extracted and purified from peripheral blood leucocytes by the salting-out procedure [32]. Fragments within the COL1A1 and MTHFR genes were amplified by polymerase chain reaction (PCR), using appropriate primers as previously described [11,12,22]. All reactions were performed in a total volume of 25 $\mathrm{mL}$ where amplification was performed using a 'hot-start' PCR protocol for the COL1A1 Sp1 and MTHFR C677T polymorphisms. Amplification of the COL1A1 $-1997 \mathrm{G} \rightarrow \mathrm{T}$ fragment was done using a hot-start/touchdown protocol starting from an annealing temperature of $60^{\circ} \mathrm{C}$ decreasing by $1{ }^{\circ} \mathrm{C}$ every two cycles until it reached $50^{\circ} \mathrm{C}$, followed by 15 cycles at an annealing temperature of $53^{\circ} \mathrm{C}$. The correct size of the amplified products was evaluated by electrophoresis of $5 \mu \mathrm{L}$ PCR product on a $1.0 \%$ agarose gel. The PCR products $(10 \mu \mathrm{L})$ were digested using the restriction enzymes MscI (COL1A1 Sp1), $B s a \mathrm{I}(\mathrm{COL} 1 \mathrm{~A} 1-1997 \mathrm{G} \rightarrow \mathrm{T}$ ) and Hinfl (MTHFR C677T) according to the manufacturer's instructions (New England Biolabs, Beverly, MA, USA). Nomenclature used for the COL1A1 $-1997 \mathrm{G} \rightarrow \mathrm{T}$ and MTHFR C677T refers to the actual nucleotide changes in the respective polymorphisms. Since the same nucleotide changes occur in both COL1A1 $-1997 \mathrm{G} \rightarrow \mathrm{T}$ and $\mathrm{Sp} 1$ polymorphisms, the $\mathrm{Sp} 1$ variant was labeled $\mathrm{SS} \rightarrow \mathrm{Ss} \rightarrow \mathrm{ss}$ (for nucleotide changes $\mathrm{GG} \rightarrow \mathrm{GT} \rightarrow \mathrm{TT}$ ). Re-analysis of $20 \%$ of samples was performed to check for accuracy of 
genotyping. No discrepancies were found from the original genotyping.

Biochemical Markers of Bone Turnover. Blood and urine samples were collected and analyzed for biochemical markers of bone turnover. Urinary deoxypyridinoline (DpD) crosslinks and serum procollagen type I were used as markers of bone resorption and formation, respectively (Metra Biosystems Inc., San Diego, CA, USA).

Bone Densitometry. Bone mineral density was measured at the LS (L2-L4) and FN using a Norland 486 Dual-Energy X-ray Absorptiometer (Norland Medical Systems Inc., New York, NY, USA). The BMD measurements were done at the Bone Density Unit, St. Luke's Hospital, G’Mangia, Malta.

Statistical Analysis. The normality of the population was tested using the non parametric Kolmogorov-Smirnov test. The chi-square test was used to test for Hardy-Weinberg and for differences in genotype frequencies between normal individuals and those with low BMD. Comparisons of continuous measurements were performed using the one-way analysis of variance (ANOVA), independent sample $t$-test or alternatively, the Kruskal-Wallis and MannWhitney tests were used for those variables not showing a normal distribution. Adjustments for age, body mass index (BMI) and years since menopause (YSM) were performed using a generalized linear model (GLM) univariate analysis of variance. Linear regression was used to test for allele dose effect. All statistical tests were performed using the Statistical Package for Social Sciences (SPSS) for Windows, student version 9.0 (SPSS, Chicago, IL, USA). The EH software by Terwillinger and Ott [33] was used to construct haplotypes of polymorphisms within the COL1A1 gene, to test for association between alleles (linkage disequilibrium) and for association of haplotypes with low BMD by comparing normal women with those who had a low BMD.

\section{RESULTS}

COL1A1 Genotypes. The distributions of the COL1A1 Sp1 and $-1997 \mathrm{G} \rightarrow \mathrm{T}$ genotypes in postmenopausal women are shown in Tables 1 and 2, respectively; both were shown to be in Hardy-

Table 1. General characteristics, lumbar and femoral bone mineral density in Maltese postmenopausal women according to COL1A1 Sp1 polymorphism.

\begin{tabular}{|c|c|c|c|c|c|c|c|}
\hline \multicolumn{4}{|c|}{ General Characteristics and Genotypes } & \multicolumn{4}{|c|}{$p$ Value $^{\mathrm{a}}$} \\
\hline & $S S$ & Ss & sS & & SS vs. Ss & SS vs. SS & Ss vs. SS \\
\hline Number & 51 & 57 & 14 & $0.75^{\mathrm{a}}$ & & & \\
\hline Percent & 41.8 & 46.7 & 11.5 & - & & & \\
\hline Age $(\text { years })^{\mathrm{b}, \mathrm{c}}$ & $55.9(7.2)$ & $55.7(6.8)$ & $55.3(8.7)$ & 0.87 & 0.73 & 0.63 & 0.74 \\
\hline $\operatorname{BMI}\left(\mathrm{kg} / \mathrm{m}^{2}\right)^{\mathrm{b}}$ & $28.3(4.4)$ & $28.7(4.1)$ & $29.5(4.5)$ & 0.62 & 0.60 & 0.36 & 0.52 \\
\hline $\begin{array}{l}\text { BMD L2-L4 }\left(\mathrm{g} / \mathrm{cm}^{2}\right)^{\mathrm{b}} \\
\text { - Adjusted }\end{array}$ & $\begin{array}{r}0.97(0.22) \\
0.97 \\
\end{array}$ & $\begin{array}{r}0.96(0.18) \\
0.96\end{array}$ & $\begin{array}{r}0.90(0.16) \\
0.89 \\
\end{array}$ & $\begin{array}{l}0.55 \\
0.26\end{array}$ & $\begin{array}{l}0.85 \\
0.64\end{array}$ & $\begin{array}{l}0.32 \\
0.11\end{array}$ & $\begin{array}{l}0.28 \\
0.17\end{array}$ \\
\hline $\begin{array}{l}\text { BMD femoral }\left(\mathrm{g} / \mathrm{cm}^{2}\right)^{b} \\
\text { - Adjusted }\end{array}$ & $\begin{array}{r}0.86(0.14) \\
0.86\end{array}$ & $\begin{array}{r}0.83(0.13) \\
0.83\end{array}$ & $\begin{array}{r}0.85(0.12) \\
0.84\end{array}$ & $\begin{array}{l}0.50 \\
0.29\end{array}$ & $\begin{array}{l}0.25 \\
0.13\end{array}$ & $\begin{array}{l}0.85 \\
0.58\end{array}$ & $\begin{array}{l}0.56 \\
0.65\end{array}$ \\
\hline $\begin{array}{l}\text { Ward's Triangle }\left(\mathrm{g} / \mathrm{cm}^{2}\right)^{\mathrm{b}} \\
\text { - Adjusted }\end{array}$ & $\begin{array}{r}0.64(0.17) \\
0.64 \\
\end{array}$ & $\begin{array}{r}0.62(0.14) \\
0.62 \\
\end{array}$ & $\begin{array}{r}0.64(0.12) \\
0.62 \\
\end{array}$ & $\begin{array}{l}0.88 \\
0.59\end{array}$ & $\begin{array}{l}0.65 \\
0.35\end{array}$ & $\begin{array}{l}0.98 \\
0.60 \\
\end{array}$ & $\begin{array}{l}0.72 \\
0.97\end{array}$ \\
\hline $\begin{array}{l}\text { Trochanter }\left(\mathrm{g} / \mathrm{cm}^{2}\right)^{\mathrm{b}} \\
\text { - Adjusted }\end{array}$ & $\begin{array}{r}0.67(0.14) \\
0.68\end{array}$ & $\begin{array}{r}0.69(0.13) \\
0.68\end{array}$ & $\begin{array}{r}0.70(0.09) \\
0.68\end{array}$ & $\begin{array}{l}0.74 \\
0.97\end{array}$ & $\begin{array}{l}0.55 \\
0.83\end{array}$ & $\begin{array}{l}0.52 \\
0.91\end{array}$ & $\begin{array}{l}0.78 \\
0.87\end{array}$ \\
\hline Procollagen $(\mathrm{ng} / \mathrm{mL})^{\mathrm{b}, \mathrm{c}}$ & $88.4(28.0)$ & $93.5(49.9)$ & $141.8(100.4)$ & 0.38 & 0.74 & 0.29 & 0.14 \\
\hline $\mathrm{DpD}(\mathrm{nmol} / \mathrm{mmol} \text { creatinine })^{\mathrm{b}}$ & $9.1(2.9)$ & $9.1(3.6)$ & $10.2(3.3)$ & 0.69 & 0.96 & 0.37 & 0.46 \\
\hline
\end{tabular}

${ }^{a} p$ Value obtained for Hardy-Weinberg Equilibrium (chi-square).

${ }^{\mathrm{b}}$ Values are means $\pm \mathrm{SD}$ in parenthesis; $p$ values obtained by ANOVA or independent sample $t$-test.

${ }^{c}$ Kruskal-Wallis or Mann-Whitney used for age and procollagen.

${ }^{\mathrm{d}}$ Values are adjusted for age, BMI, years since menopause (YSM); 95\% confidence interval (CI) given by univariate analysis (not shown). 
Weinberg equilibrium $(p>0.05)$. Allele frequencies for the Sp1 polymorphism were $65.2 \%$ for the $\mathrm{S}$ allele and $34.8 \%$ for the s, with a heterozygosity rate of $45.6 \%$. The calculated heterozygosity for the $-1997 \mathrm{G} \rightarrow \mathrm{T}$ polymorphism was $38.3 \%$, with a frequency of $25.6 \%$ for the $\mathrm{T}$ and $74.4 \%$ for the $\mathrm{G}$ allele. Linkage disequilibrium was observed between these two polymorphisms $[\chi 2=12.45$; $p=0.01$; degrees of freedom $(\mathrm{df})=3]$ with the combined genotype $\mathrm{GG} \rightarrow \mathrm{Ss}$ occurring in $33.6 \%$. When constructing haplotypes, the frequencies of the most common haplotypes were $41.5 \%$ for $\mathrm{G} \rightarrow \mathrm{S}$ and $23.2 \%$ for $\mathrm{T} \rightarrow \mathrm{S}$.

Both polymorphisms in the promoter region of the COL1A1 gene did not show any influence on the LS and FN BMD (Tables 1 and 2). Statistical significance was not reached on tests for genetic models of a dominant or recessive allele (Table 3) or for an allele dose effect by linear regression ( $p$ $>0.05$ ) (data not shown).

MTHFR C677T Polymorphism. The genotype frequencies for the MTHFR C677T polymorphism observed in postmenopausal women are shown in Table 4. Allele frequencies observed were $63.9 \%$ for the $\mathrm{C}$ allele and $36.1 \%$ for the $\mathrm{T}$, yielding a calculated heterozygosity rate of $46.3 \%$.

The LS, FN, trochanter and Ward's triangle BMD were lowest in CC homozygotes, although the differences between the genotypes did not reach statistical significance $(p>0.05)$ (Table 4$)$. The $C$ allele was significantly correlated with trochanter BMD and, to a lesser degree, with FN BMD (Table 5 ). No allele dose effect was observed at any anatomical site for the same allele $(p>0.05)$.

There were no differences in the distribution of genotype frequencies of both COL1A1 polymorphisms between normal postmenopausal women (independent sample $t$-score $>-1.0$ ) and those with a low LS and/or FN BMD when tested by chi-square $(p>0.05)$ (Table 6). Haplotype frequencies were not statistically significant when compared between these two groups $(\chi 2=1.14 ; p=0.76 ; \mathrm{df}=3)$ (Table 6). The MTHFR C677T genotype frequencies did not differ significantly between the normal women and those with a low BMD $(\chi 2=0.78 ; p=0.68$; $\mathrm{df}=2)($ Table 6).

The MTHFR C677T Frequency in the Maltese Population at Birth. In neonates $(n=245)$,

Table 2. General characteristics, lumbar and femoral bone mineral density in Maltese postmenopausal women according to COL1A1 $-1997 \mathrm{G} \rightarrow$ T polymorphism.

\begin{tabular}{|c|c|c|c|c|c|c|c|}
\hline \multicolumn{4}{|c|}{ General Characteristics and Genotypes } & \multicolumn{4}{|c|}{$p$ Value $^{\mathrm{a}}$} \\
\hline & TT & TG & GG & & TT vs. TG & TT vs. GG & TGvs. GG \\
\hline Number & 10 & 43 & 70 & $0.36^{\mathrm{a}}$ & & & \\
\hline Percent & 8.1 & 35.0 & 56.9 & - & & & \\
\hline Age $\left(\right.$ years) ${ }^{\mathrm{b}, \mathrm{c}}$ & $57.3(6.1)$ & $55.6(6.9)$ & $55.5(7.5)$ & 0.50 & 0.27 & 0.25 & 0.88 \\
\hline BMI $\left(\mathrm{kg} / \mathrm{m}^{2}\right)^{\mathrm{b}}$ & $27.3(5.0)$ & $28.9(4.5)$ & $28.4(3.9)$ & 0.60 & 0.38 & 0.47 & 0.55 \\
\hline $\begin{array}{l}\text { BMD L2-L4 }\left(\mathrm{g} / \mathrm{cm}^{2}\right)^{\mathrm{b}} \\
\text { - } \text { Adjusted }^{\mathrm{d}}\end{array}$ & $\begin{array}{r}0.97(0.16) \\
1.01\end{array}$ & $\begin{array}{r}0.97(0.22) \\
0.97\end{array}$ & $\begin{array}{r}0.94(0.18) \\
0.94\end{array}$ & $\begin{array}{l}0.67 \\
0.37\end{array}$ & $\begin{array}{l}0.98 \\
0.46\end{array}$ & $\begin{array}{l}0.58 \\
0.24\end{array}$ & $\begin{array}{l}0.41 \\
0.34\end{array}$ \\
\hline $\begin{array}{l}\text { BMD femoral }\left(\mathrm{g} / \mathrm{cm}^{2}\right)^{\mathrm{b}} \\
\text { - } \text { Adjusted }^{\mathrm{d}}\end{array}$ & $\begin{array}{r}0.85(0.15) \\
0.88\end{array}$ & $\begin{array}{r}0.85(0.14) \\
0.85\end{array}$ & $\begin{array}{r}0.83(0.13) \\
0.83\end{array}$ & $\begin{array}{l}0.83 \\
0.43\end{array}$ & $\begin{array}{l}0.91 \\
0.41\end{array}$ & $\begin{array}{l}0.67 \\
0.24\end{array}$ & $\begin{array}{l}0.60 \\
0.49\end{array}$ \\
\hline $\begin{array}{l}\text { Ward's Triangle }\left(\mathrm{g} / \mathrm{cm}^{2}\right)^{\mathrm{b}} \\
\text { - Adjusted }\end{array}$ & $\begin{array}{r}0.64(0.15) \\
0.69 \\
\end{array}$ & $\begin{array}{r}0.63(0.16) \\
0.63 \\
\end{array}$ & $\begin{array}{r}0.62(0.14) \\
0.62 \\
\end{array}$ & $\begin{array}{l}0.90 \\
0.45 \\
\end{array}$ & $\begin{array}{l}0.89 \\
0.31 \\
\end{array}$ & $\begin{array}{l}0.72 \\
0.21\end{array}$ & $\begin{array}{l}0.71 \\
0.75\end{array}$ \\
\hline $\begin{array}{l}\text { Trochanter }\left(\mathrm{g} / \mathrm{cm}^{2}\right)^{\mathrm{b}} \\
\text { - Adjusted }\end{array}$ & $\begin{array}{r}0.64(0.15) \\
0.67\end{array}$ & $\begin{array}{r}0.68(0.14) \\
0.68 \\
\end{array}$ & $\begin{array}{r}0.68(0.12) \\
0.67 \\
\end{array}$ & $\begin{array}{r}0.71 \\
096\end{array}$ & $\begin{array}{l}0.45 \\
0.80\end{array}$ & $\begin{array}{l}0.46 \\
0.93\end{array}$ & $\begin{array}{l}0.77 \\
0.83\end{array}$ \\
\hline Procollagen $(\mathrm{ng} / \mathrm{mL})^{\mathrm{b}, \mathrm{c}}$ & $88.6(26.8)$ & $103.9(86.1)$ & $93.0(45.4)$ & 0.91 & 0.85 & 0.74 & 0.74 \\
\hline $\mathrm{DpD}(\mathrm{nmol} / \mathrm{mmol} \text { creatinine })^{\mathrm{b}}$ & $8.3(2.2)$ & $9.6(3.4)$ & $9.1(3.3)$ & 0.66 & 0.39 & 0.57 & 0.57 \\
\hline
\end{tabular}

${ }^{a} p$ Value obtained for Hardy-Weinberg Equilibrium (chi-square).

${ }^{\mathrm{b}}$ Values are means \pm SD in parenthesis; $p$ values obtained by ANOVA or independent sample $t$-test.

${ }^{c}$ Kruskal-Wallis or Mann-Whitney used for age and procollagen.

${ }^{\mathrm{d}}$ Values are adjusted for age, BMI, YSM; 95\% CI given by univariate analysis (not shown). 
Table 3. Effects of COL1A1 alleles on bone mineral density in postmenopausal women assuming genetic models of a dominant or recessive allele.

\begin{tabular}{|c|c|c|c|c|c|c|}
\hline & \multicolumn{3}{|c|}{ Dominant Modela $^{\mathbf{a}}$} & \multicolumn{3}{|c|}{ Recessive Model $^{\text {a }}$} \\
\hline & SS & Ss and ss & $p$ Value $^{\mathrm{b}}$ & SS and Ss & ss & $p$ Value $^{\mathrm{b}}$ \\
\hline Number & 49 & 71 & - & 106 & 14 & - \\
\hline BMD L2-L4 $\left(\mathrm{g} / \mathrm{cm}^{2}\right)^{\mathrm{c}}$ & $0.97(0.22)$ & $0.95(0.17)$ & 0.61 & $0.96(0.20)$ & $0.90(0.16)$ & 0.28 \\
\hline - Adjusted $^{\mathrm{d}}$ & 0.97 & 0.94 & 0.37 & 0.96 & 0.89 & 0.11 \\
\hline BMD femoral $\left(\mathrm{g} / \mathrm{cm}^{2}\right)^{\mathrm{c}}$ & $0.86(0.14)$ & $0.83(0.13)$ & 0.30 & $0.84(0.14)$ & $0.85(0.12)$ & 0.83 \\
\hline - Adjusted $^{\mathrm{d}}$ & 0.86 & 0.83 & 0.13 & 0.84 & 0.84 & 0.98 \\
\hline Ward's Triangle $\left(\mathrm{g} / \mathrm{cm}^{2}\right)^{\mathrm{c}}$ & $0.64(0.17)$ & $0.62(0.13)$ & 0.69 & $0.63(0.15)$ & $0.64(0.12)$ & 0.84 \\
\hline - Adjusted ${ }^{\mathrm{d}}$ & 0.64 & 0.62 & 0.31 & 0.63 & 0.62 & 0.79 \\
\hline Trochanter $\left(\mathrm{g} / \mathrm{cm}^{2}\right)^{\mathrm{c}}$ & $0.67(0.14)$ & $0.69(0.12)$ & 0.47 & $0.68(0.13)$ & $0.70(0.09)$ & 0.64 \\
\hline - Adjusted ${ }^{\mathrm{d}}$ & 0.68 & 0.68 & 0.83 & 0.68 & 0.68 & 0.94 \\
\hline$-1997 T \rightarrow G$ Polymorphism & $T T$ & $T G$ and $G G$ & $p$ Value $^{\mathrm{b}}$ & $T T$ and $T G$ & $G G$ & $p$ Value $^{\mathrm{b}}$ \\
\hline Number & 10 & 113 & - & 53 & 70 & - \\
\hline BMD L2-L4 $\left(\mathrm{g} / \mathrm{cm}^{2}\right)^{\mathrm{c}}$ & $0.97(0.16)$ & $0.95(0.20)$ & 0.74 & $0.97(0.21)$ & $0.94(0.18)$ & 0.37 \\
\hline - Adjusted $^{\mathrm{d}}$ & 1.01 & 0.95 & 0.31 & 0.98 & 0.94 & 0.21 \\
\hline BMD femoral $\left(\mathrm{g} / \mathrm{cm}^{2}\right)^{\mathrm{c}}$ & $0.85(0.15)$ & $0.84(0.14)$ & 0.75 & $0.85(0.14)$ & $0.83(0.13)$ & 0.55 \\
\hline - Adjusted $^{\mathrm{d}}$ & 0.88 & 0.84 & 0.28 & 0.85 & 0.83 & 0.30 \\
\hline Ward's Triangle $\left(\mathrm{g} / \mathrm{cm}^{2}\right)^{\mathrm{c}}$ & $0.64(0.15)$ & $0.63(0.15)$ & 0.78 & $0.63(0.14)$ & $0.62(0.14$ & 0.66 \\
\hline - Adjusted ${ }^{\mathrm{d}}$ & 0.69 & 0.62 & 0.22 & 064 & 0.62 & 0.49 \\
\hline Trochanter $\left(\mathrm{g} / \mathrm{cm}^{2}\right)^{\mathrm{c}}$ & $0.64(0.15)$ & $0.68(0.13)$ & 0.44 & $0.68(0.14)$ & $0.68(0.12)$ & 0.96 \\
\hline - Adjusted $^{\mathrm{d}}$ & 0.67 & 0.68 & 0.88 & 0.68 & 0.66 & 0.86 \\
\hline
\end{tabular}

${ }^{a}$ Mean BMD compared between genotype groups assuming hypothesis: A) dominant S or T alleles; B) recessive S or T alleles.

${ }^{\mathrm{b}} p$ Values obtained by unpaired independent sample $t$-test (two-tailed).

${ }^{\mathrm{c}}$ Values are means $\pm \mathrm{SD}$ in parenthesis.

${ }^{\mathrm{d}}$ Values are adjusted for age, BMI, YSM; 95\% CI given by univariate analysis (not shown).

Table 4. General characteristics, lumbar and femoral bone mineral density in Maltese postmenopausal women according to the MTHFR C677T polymorphism.

\begin{tabular}{|c|c|c|c|c|c|c|c|}
\hline \multicolumn{4}{|c|}{ General Characteristics and Genotypes } & \multicolumn{4}{|c|}{$p$ Value $^{\mathrm{a}}$} \\
\hline & $C C$ & $C T$ & $T T$ & & CC vs. CT & CC vs. TT & CT vs. TT \\
\hline Number & 49 & 63 & 14 & $0.35^{\mathrm{a}}$ & & & \\
\hline Percent & 38.9 & 50.0 & 11.1 & - & & & \\
\hline Age $(\text { years })^{b, c}$ & $56.8(7.6)$ & $55.7(6.9)$ & $61.1(4.4)$ & 0.03 & 0.51 & 0.01 & 0.02 \\
\hline $\operatorname{BMI}\left(\mathrm{kg} / \mathrm{m}^{2}\right)^{\mathrm{b}}$ & $29.2(4.8)$ & $28.2(3.6)$ & $28.2(4.6)$ & 0.46 & 0.25 & 0.52 & 0.98 \\
\hline BMD L2-L4 $\left(\mathrm{g} / \mathrm{cm}^{2}\right)^{\mathrm{b}}$ & $0.94(0.22)$ & $0.96(0.18)$ & $0.97(0.12)$ & 0.72 & 0.48 & 0.57 & 0.85 \\
\hline - Adjusted $^{\mathrm{d}}$ & 0.94 & 0.97 & 0.94 & 0.57 & 0.31 & 0.68 & 0.66 \\
\hline BMD femoral $\left(\mathrm{g} / \mathrm{cm}^{2}\right)^{\mathrm{b}}$ & $0.82(0.14)$ & $0.85(0.13)$ & $0.89(0.13)$ & 0.12 & 0.13 & 0.08 & 0.35 \\
\hline - Adjusted $^{\mathrm{d}}$ & 0.82 & 0.86 & 0.86 & 0.14 & 0.07 & 0.23 & 0.87 \\
\hline Ward's Triangle $\left(\mathrm{g} / \mathrm{cm}^{2}\right)^{\mathrm{b}}$ & $0.60(0.16)$ & $0.64(0.14)$ & $0.68(0.09)$ & 0.25 & 0.22 & 0.18 & 0.43 \\
\hline - Adjusted ${ }^{\mathrm{d}}$ & 0.61 & 0.64 & 0.63 & 0.45 & 0.24 & 0.56 & 0.89 \\
\hline Trochanter $\left(\mathrm{g} / \mathrm{cm}^{2}\right)^{\mathrm{b}}$ & $0.65(0.14)$ & $0.69(0.12)$ & $0.74(0.08)$ & 0.10 & 0.15 & 0.07 & 0.19 \\
\hline - Adjusted ${ }^{\mathrm{d}}$ & 0.65 & 0.69 & 0.71 & 0.08 & 0.04 & 0.22 & 0.55 \\
\hline Procollagen $(\mathrm{ng} / \mathrm{mL})^{\mathrm{b}, \mathrm{c}}$ & $95.1(28.9)$ & $99.7(82.6)$ & $82.8(29.5)$ & 0.21 & 0.08 & 0.43 & 0.88 \\
\hline $\mathrm{DpD}\left(\mathrm{nmol} / \mathrm{mmol}\right.$ creatinine) ${ }^{\mathrm{b}}$ & $9.6(3.6)$ & $8.8(3.0)$ & $9.3(3.1)$ & 0.62 & 0.34 & 0.87 & 0.67 \\
\hline
\end{tabular}

${ }^{\mathrm{a}} p$ Value obtained for Hardy-Weinberg Equilibrium (chi-square).

${ }^{\mathrm{b}}$ Values are means \pm SD in parenthesis; $p$ values obtained by ANOVA or independent sample t-test;

${ }^{c}$ Kruskal-Wallis or Mann-Whitney used for age and procollagen.

${ }^{\mathrm{d}}$ Values are adjusted for age, BMI, YSM; 95\% CI given by univariate analysis (not shown). 
Table 5. Effects of the MTHFR alleles on bone mineral density in postmenopausal women assuming genetic models of a dominant or recessive allele.

\begin{tabular}{|c|c|c|c|c|c|c|}
\hline \multirow[b]{2}{*}{ C6776 Polymorphism } & \multicolumn{3}{|c|}{ 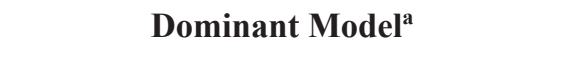 } & \multicolumn{3}{|c|}{ Recessive Model $^{\text {a }}$} \\
\hline & $\mathrm{CC}$ & $\mathrm{CC}$ and TT & $p$ Value $^{\mathrm{b}}$ & $C C$ and $C T$ & $T T$ & $p$ Value $^{\mathrm{b}}$ \\
\hline Number & 47 & 77 & - & 110 & 14 & - \\
\hline $\begin{array}{l}\text { BMD L2-L4 }\left(\mathrm{g} / \mathrm{cm}^{2}\right)^{\mathrm{c}} \\
\text { - Adjusted }\end{array}$ & $\begin{array}{c}0.94(0.22) \\
0.94\end{array}$ & $\begin{array}{c}0.96(0.17) \\
0.96\end{array}$ & $\begin{array}{l}0.42 \\
0.42\end{array}$ & $\begin{array}{c}0.95(0.20) \\
0.96\end{array}$ & $\begin{array}{c}0.97(0.12) \\
0.93\end{array}$ & $\begin{array}{l}0.71 \\
0.64\end{array}$ \\
\hline $\begin{array}{l}\text { BMD femoral }\left(\mathrm{g} / \mathrm{cm}^{2}\right)^{\mathrm{c}} \\
\text { - } \text { Adjusted }^{\mathrm{d}}\end{array}$ & $\begin{array}{c}0.81(0.14) \\
0.82\end{array}$ & $\begin{array}{c}0.86(0.13) \\
0.86\end{array}$ & $\begin{array}{l}0.07 \\
0.05\end{array}$ & $\begin{array}{c}0.84(0.14) \\
0.84\end{array}$ & $\begin{array}{c}0.89(0.13) \\
0.86\end{array}$ & $\begin{array}{l}0.18 \\
0.49\end{array}$ \\
\hline $\begin{array}{l}\text { Ward's Triangle }\left(\mathrm{g} / \mathrm{cm}^{2}\right)^{\mathrm{c}} \\
\text { - Adjusted }\end{array}$ & $\begin{array}{c}0.60(0.16) \\
0.61\end{array}$ & $\begin{array}{c}0.64(0.14) \\
0.64\end{array}$ & $\begin{array}{l}0.14 \\
0.22\end{array}$ & $\begin{array}{c}0.62(0.15) \\
0.63\end{array}$ & $\begin{array}{c}0.68(0.09) \\
0.63\end{array}$ & $\begin{array}{l}0.29 \\
0.87\end{array}$ \\
\hline $\begin{array}{l}\text { Trochanter }\left(\mathrm{g} / \mathrm{cm}^{2}\right)^{\mathrm{c}} \\
\text { - Adjusted }\end{array}$ & $\begin{array}{c}0.65(0.14) \\
0.65 \\
\end{array}$ & $\begin{array}{c}0.70(0.11) \\
0.70 \\
\end{array}$ & $\begin{array}{l}0.07 \\
0.03\end{array}$ & $\begin{array}{c}0.67(0.13) \\
0.67\end{array}$ & $\begin{array}{c}0.74(0.08) \\
0.71\end{array}$ & $\begin{array}{l}0.12 \\
0.33 \\
\end{array}$ \\
\hline
\end{tabular}

${ }^{\text {a }}$ Mean BMD compared between genotype groups assuming hypothesis: A) dominant $\mathrm{C}$ allele; $\mathrm{B}$ ) recessive $\mathrm{C}$ allele.

${ }^{\mathrm{b}} p$ Values obtained by unpaired independent sample $t$-test (two-tailed).

${ }^{\mathrm{c}}$ Values are means $\pm \mathrm{SD}$ in parenthesis.

${ }^{\mathrm{d}}$ Values are adjusted for age, BMI, YSM; 95\% CI given by univariate analysis (not shown).

Table 6. Genotype and haplotype frequencies (\%) in normal postmenopausal women and women having a low bone mineral density.

\begin{tabular}{|l|r|r|c|}
\hline & Normal & $\begin{array}{r}\text { Low } \\
\text { BMD }\end{array}$ & p Value \\
\hline COL1A1 Sp1 & 46.9 & 36.6 & \\
$\bullet$ SS & 42.9 & 50.7 & 0.53 \\
$\bullet$ Ss & 10.2 & 12.7 & \\
$\bullet$ ss & & & \\
\hline COL1A1-1997 & 10.0 & 5.6 & \\
G $\rightarrow$ T & 36.0 & 33.8 & 0.60 \\
$\bullet$ TT & 54.0 & 60.6 & \\
$\bullet$ TG & & & \\
$\bullet$ GG & 33.3 & 41.1 & \\
\hline MTHFR & 54.9 & 47.9 & 0.68 \\
$\bullet$ CC & 11.8 & 11.0 & \\
$\bullet$ CT & & & \\
$\bullet$ TT & 25.6 & 21.0 & \\
\hline COL1A1 Haplotype & 42.1 & 40.6 & \multirow{2}{*}{0.76} \\
$\bullet$ T/S & 1.5 & 2.2 & \\
$\bullet$ G/S & 30.8 & 36.2 & \\
$\bullet$ T/s & & & \\
$\bullet$ G/s & & & \\
\hline
\end{tabular}

${ }^{a} p$ Values obtained by the chi-squared test.

genotype frequencies for this polymorphism were 46.1\% CC, 43.3\% CT and 10.6\% TT and were in Hardy-Weinberg equilibrium $(\chi 2=0.02 ; p=0.88$; $\mathrm{df}=1)$.

\section{DISCUSSION}

Genotype frequencies observed for the -1997 $\mathrm{G} \rightarrow \mathrm{T}$ polymorphism in our group of postmenopausal women were similar to those reported from Spain [22]. Those for the Sp1 polymorphism were closer to those in two Southern European populations [20,34] than in other Caucasians [11,16,22,35-37]. The Sp1 polymorphism is absent in Asian populations [38,39].

For the Sp1 polymorphism the highest LS BMD was found in SS homozygotes, while the influence on FN BMD was minimal but the differences did not reach significance for both sites. This trend agrees with that reported by Garnero and co-workers [40] who found SS homozygotes to have the highest and statistically significant LS BMD, but no association with FN BMD. An influence of Sp1 polymorphism on LS BMD was observed by others [11,35] and also an association with FN BMD [37]. Other investigators did not find the $\mathrm{Sp} 1$ polymorphism to affect BMD $[21,22]$ or found it to be associated with an increased fracture risk at the LS, FN and other skeletal sites $[17,20,36]$. The increased risk of fracture at the FN has been explained by differences in FN geometry between different genotypes [41].

Our results for the $-1997 \mathrm{G} \rightarrow \mathrm{T}$ SNP differed from those observed in Spain [22], where the lowest BMD was found in TT, and not in GG homozygotes, in contrast to our results. As reported 
in the Spanish [22] and American [42] populations, strong linkage disequilibrium between the -1997 $\mathrm{G} \rightarrow \mathrm{T}$ and $\mathrm{Sp} 1$ polymorphism was also observed in our study, although it do not seem to affect BMD. It has been suggested [22] that binding of osteoblast nuclear factors may be affected by the $-1997 \mathrm{G} \rightarrow \mathrm{T}$ nucleotide change in the COL1A1 gene since the $\mathrm{G}$ allele has a higher binding capacity to singlestranded binding proteins. Polymorphisms within the promoter region act in synergy with the Sp1 SNP to affect binding capacities of transcriptional factors such as Nmp4/CIZ [43]. In a recent study the haplotype of these two polymorphisms was shown to have a greater effect on BMD than when analyzed separately, suggesting that interactions between the Sp1 and -1997 polymorphisms may affect the phenotype [42]. The binding affinity of Sp1 may be influenced by other nearby polymorphisms, including the $-1997 \mathrm{G} \rightarrow \mathrm{T}$, and result in an increased expression of COL1A1 in relation to COL1A2, thus affecting bone quality [42].

At the phenotypic level, these polymorphisms can be influenced by interactions with other genes and with various environmental factors. We analyzed a common C677T polymorphism within the MTHFR gene for any correlation with BMD and found that TT homozygotes had the highest, but not statistically significant BMD at all anatomical sites, and that the $\mathrm{C}$ allele had a negative effect on trochanter BMD. This contrasts with what was reported on Japanese women where the $\mathrm{T}$ allele was associated with low BMD [27]. However, in a Danish study the $\mathrm{T}$ allele was associated with a decreased risk of fractures independently of BMD [12]. A degree of genotypic and allelic variations exist among ethnic groups such that there is a higher prevalence of TT homozygotes in southern European populations, Hispanics and Mexicans [44]. The frequency of TT homozygotes we observed is similar to that reported for Spanish, French [44] and Portuguese [45] populations, but lower than that for Italians [44] and Japanese [27], and higher than that for northern Europeans $[12,44]$.

One reason for these conflicting results may be differences in folate intake in these populations. Recently, TT homozygotes were found to be associated with low BMD only in the presence of low plasma folate concentration, whereas the highest femoral and trochanter BMD were found when plasma folate concentrations were above $4 \mathrm{ng} /$ $\mathrm{mL}$ [24]. It has been suggested that the north-south increase in the prevalence of TT homozygotes may be influenced by the higher folic acid content of the Mediterranean diet [46]. Low plasma folate may result in hyperhomocysteinemia, independently of the C677T polymorphism [47]. Levels of riboflavin and vitamins $\mathrm{B}_{12}$ and $\mathrm{B}_{6}$ also modify the effect of the MTHFR TT genotype on BMD in the Danish population, although it was estimated that only $2 \%$ of the population will benefit from vitamin B supplementation [48].

The C677T SNP may also be influenced by nearby polymorphisms that are in linkage disequilibrium with the C677T SNP, an example being the $1298 \mathrm{~A} \rightarrow \mathrm{C}$ that is known to affect plasma homocysteine levels [45]. Different phenotypic expression of this polymorphism might be affected by other variants within nearby genes on the same candidate region $1 \mathrm{p} 36[13,9]$, as well as by genes coding for enzymes involved in the same metabolic pathway as methionine synthase reductase and cystathionine synthase. The C677T polymorphism in the MTHFR gene is also known to affect DNA methylation, which is a major epigenetic controller of gene expression. It has been reported that TT homozygotes had a diminished level of DNA methylation when compared to $\mathrm{CC}$ homozygotes only when folate levels were low [49].

We found no association between two COL1A1 gene variants and an increased risk for low BMD in Maltese postmenopausal women. It is clear that allelic heterogeneity exists between different populations, and that different genes and gene variants may be responsible for osteoporosis in different ethnic groups. Future studies should focus on gene expression and how this may affect the phenotype in relation to the environment and other epigenetic mechanisms that play important roles in gene regulation and in proteinomics.

\section{REFERENCES}

1. Huang QY, Recker RR, Deng HW. Searching for osteoporosis genes in the post-genome era: progress and challenges. Osteoporos Int 2003; 14(9): 701-715.

2. Liu YZ, Liu YJ, Recker RR, Deng HW. Molecular studies of identification of genes for 
osteoporosis: the 2002 update. J Endocrinol 2003; 177(2): 147-196.

3. Zmuda JM, Cauley JA, Ferrell RE. Molecular epidemiology of vitamin D receptor gene variants. Endocrinol Rev 2000; 22(2): 203-217.

4. Ionnidis JPA, Stavrou I, Trikalinos TA, Zois C, Brandi ML, Gennari L, Albagha O, Ralston SH, Tsatsoulis A. Association of polymorphisms of the estrogen receptor $\alpha$ gene with bone mineral density and fracture risk in women: a meta-analysis. J Bone Miner Res 2002; 17(11): 2048-2060.

5. Urano T, Shiraki M, Ezura Y, Fujita M, Sekine E, Hoshino S, Hosoi T, Orimo H, Emi M, Ouchi Y, Inoue S. Association of a single nucleotide polymorphism in low density lipoprotein receptorrelated protein 5 gene with bone mineral density. J Bone Miner Metab 2004; 22(4): 341-345.

6. Langdahl BL, Carstens M, Stenkjaer L, Eriksen EF. Polymorphisms in the osteoprotegerin gene are associated with osteoporotic fractures. J Bone Miner Res 2002; 17(7): 1245-1255.

7. Chung HW, Seo JS, Hur SE, Kim HL, Kim JY, Jung JH, Kim LH, Park BL, Shin HD. Association of interleukin- 6 promoter variant with bone mineral density in pre-menopausal women. J Hum Genet 2003; 48(5): 243-248.

8. Langdahl BL, Carstens M, Stenkjaer L, Eriksen EF. Polymorphisms in the transforming growth factor $\beta 1$ gene and osteoporosis. Bone 2003; 32(3): 297-310.

9. Kim JG, Roh KR, Lee JY. The relationship among serum insulin-like growth factor-I, insulinlike growth factor-I gene polymorphism, and bone mineral density in postmenopausal women in Korea. Am J Obstet Gynecol 2002; 186(3): 345-350.

10. Styrkarsdottir U, Cazier JB, Kong A, Rolfsson O, Larsen H, Bjarnadottir E, Johannsdottir VD, Sigurdadottir MS, Bagger Y, Christiansen C, Reynisdottir I, Grant SFA, Jonasson K, Frigge ML, Gulcher JR, Sigurdsson G, Stefansson K. Linkage of osteoporosis to chromosome 20p12 and association to BMP2. PLoS Biol 2003; 1(1): 1-10.

11. Grant FA, Reid DM, Blake G, Herd R, Fogelman I, Ralston SH. Reduced bone density and osteoporosis associated with a polymorphic Sp1 binding site in the Collagen type $1 \alpha 1$ gene. Nat Genet 1996; 14(2): 203-205.

12. Jorgensen HL, Madsen JS, Madsen B, Saleh MMA, Abrahamsen B, Fenger M, Lauritzen JB. Association of a common allelic polymorphism
(C677T) in the methylene tetrahydrofolate reductase gene with reduced risk of osteoporotic fractures. A case control study in Danish postmenopausal women. Calcif Tissue Int 2002; 71(5): 386-392.

13. Spotila LD, Rodriguez $\mathrm{H}$, Koch $\mathrm{M}$, Tenenhouse HS, Tenenhouse A, Li H, Devoto M. Association analysis of bone mineral density and single nucleotide polymorphisms in two candidate genes on chromosome 1p36. Calcif Tissue Int 2003; 73(2): 140-146.

14. Dalgleish R. The human type I collagen mutation database. Nucleic Acids Res 1997; 25(1): 181-187.

15. Myllyharju J, Kivirikko KI. Collagens, modifying enzymes and their mutations in humans, flies and worms. Trends Genet 2004; 20(1): 33-43.

16. MacDonald HM, McGuigan FA, New SA, Campbell MK, Golden MHN, Ralston SH, Reid DM. COL1A1 Sp1 polymorphism predicts perimenopausal and early postmenopausal spinal bone loss. J Bone Miner Res 2001; 16(9): 16341641.

17. Mann V, Ralston SH. Meta-analysis of COLIA1 Sp1 polymorphism in relation to bone mineral density and osteoporotic fracture. Bone 2003; 32(6): 711-717.

18. Brown MA, Haughton MA, Grant SFA, Gunnell AS, Henderson NK, Eisman JA. Genetic control of bone density and turnover: role of the Collagen $1 \alpha 1$, oestrogen receptor, and vitamin D receptor genes. J Bone Miner Res 2001; 16(4): 758764.

19. Mann V, Hobson EE, Li B, Stewart TL, Grant SFA, Robins SP, Aspden RM, Ralston SH. A COLIA1 Sp1 binding site polymorphism predisposes to osteoporotic fracture by affecting bone density and quality. J Clin Invest 2001; 107(7): 899-907.

20. Bernad M, Martinez ME, Escalona M, Gonzalez ML, Gonzalez C, Garces MV, DelCampo MT, Mola EM, Madero R, Carreno L. Polymorphism in the type I Collagen (COLIA1) gene and risk fractures in postmenopausal women. Bone 2002; 30(1): 223-228.

21, Aerssens J, Dequeker J, Peeters J, Breemans $\mathrm{S}$, Broos P, Boonen S. Polymorphisms of the VDR, ER and COLIA1 genes and osteoporotic hip fracture in elderly postmenopausal women. Osteoporos Int 2000; 11(7): 583-591.

22. Garcia-Giralt N, Nogues X, Enjuanes A, Puig J, Mellibovski L, Bay-Jensen A, Carreras R, 
Balcells S, Diez-Perez A, Grinberg D. Two new single nucleotide polymorphisms in the COLIA1 upstream regulatory region and their relationship to bone mineral density. J Bone Miner Res 2002; 17(3): 384-393.

23. Lubec B, Fang-Kircher S, Lubec T, Blom $\mathrm{HJ}$, Boers GH. Evidence for McKusick's hypothesis of deficient collagen cross-linking in patients with homocystinuria. Biochim Biophys Acta 1996; 1315(3): 159-162.

24. McLean RR, Karasik D, Selhub J, Tucker KL, Ordovas JM, Russo GT, Cupples LA, Jacques PF, Kiel DP. Association of a common polymorphism in the methylenetetrahydrofolate reductase (MTHFR) gene with bone phenotypes depends on plasma folate status. J Bone Miner Res 2003; 19(3): 410418.

25. Kang SS, Wong PW, Zhou JM, Sora J, Lessick M, Ruggie N, Grcevich G. Thermolabile methylenetetrahydrofolate reductase in patients with coronary artery disease. Metabolism 1988; 37(7): 611-613.

26. Van der Put NMJ, Gabreels F, Stevens EMB, Smeitink JAM, Trijbels FJM, Eskes TKAB, Van den Heuvel LP, Blom HJ. A second common mutation in the methylenetetrahydrofolate reductase gene: an additional risk factor for neural tube defects? Am J Hum Genet 1998; 62(5): 1044-1051.

27. Miyao M, Morita $H$, Kurihara $H$, Inoue S, Hoshino S, Shiraki M, Yazaki Y, Ouchi Y. Association of methylenetetrahydrofolate reductase (MTHFR) polymorphism with bone mineral density in postmenopausal Japanese women. Calcif Tissue Int 2000; 66(3): 190-194.

28. Devoto M, Shimoya K, Caminis J, Ott J, Tenenhouse A, Whyte MP, Sereda L, Hall S, Considine E, Williams CJ, Tromp G, Kuivaniemi H, Ala-Kokko L, Prockop DJ, Spotila LD. First-stage autosomal genome screen in extended pedigrees suggests genes predisposing to low bone mineral density on chromosomes 1p, 2p and 4q. Eur J Hum Genet 1998; 6(2): 151-157.

29. Devoto M, Specchia C, Li HH, Caminis J, Tenenhouse A, Rodriguez H, Spotila LD. Variance component linkage analysis indicates a QTL for femoral neck bone mineral density on chromosome 1p36. Hum Mol Genet 2001; 10(21): 2447-2452.

30. Wilson SG, Reed PW, Bansal A, Chiano M, Lindersson M, Langdown M, Prince RL, Thompson D, Thompson E, Bailey M, Kleyn PW, Sambrook P,
Shi MM, Spector TD. Comparison of genome screens for two independent cohorts provides replication of suggestive linkage of bone mineral density to $3 \mathrm{p} 21$ and 1p36. Am J Hum Genet 2003; 72(1): 144-155.

31. Kanis JA, Melton LJ, Christiansen C, Johnston CC, Khaltaev N. The diagnosis of osteoporosis. J Bone Miner Res 1994; 9(8): 11371141.

32. Miller SA, Dykes DD, Polesky HF. A simple salting out procedure for extracting DNA from human nucleated cells. Nucleic Acids Res 1988; 16(3): 1215.

33. Terwillinger JD, OttJ.Linkage disequilibrium between alleles at marker loci. In: Terwillinger JD, OTT J, Eds. Handbook of Human Genetic Linkage. Baltimore: John Hopkins University Press. 1994; 188-198.

34. Braga V, Mottes M, Mirandola S, Lisi V, Malerba G, Sartori L, Bianchi G, Gatti D, Rossini M, Bianchini D, Adami S. Association of CTR and COL1A1 alleles with BMD values in peri- and postmenopausal women. Calcif Tissue Int 2000; 67(5): 361-366.

35. Efstathiadou Z, Kranas V, Ionnnidis JPA, Georgiou I, Tsatsoulis A. The Sp1 COL1A1 gene polymorphism, and not vitamin $\mathrm{D}$ receptor or estrogen receptor gene polymorphisms, determines bone mineral density in postmenopausal Greek women. Osteoporos Int 2001; 12(4): 326-331.

36. Weichetova M, Stepan JJ, Michalska A, Haas T, Pols HAP, Uitterlinden AG. COL1A1 polymorphism contributes to bone mineral density to assess prevalent wrist fractures. Bone 2000; 26(3): 287-290.

37. Gerdhem P, Brandstrom H, Stiger F, Obrant $\mathrm{K}$, Melhus $\mathrm{H}$, Ljunggren $\mathrm{O}$, Kindmark A, Akesson K. Association of Collagen type 1 (COL1A1) Sp1 binding site polymorphism to femoral neck bone mineral density and wrist fracture in 1044 elderly Swedish women. Calcif Tissue Int 2004; 74(3): 264269.

38. Lei SF, Deng FY, Liu XH, Huang QR, Qin Y, Zhou Q, Jiang DK, Li YM, Mo XY, Liu MY, Chen XD, Wu XS, Shen H, Dvornyk V, Zhao L, Recker RR, Deng HW. Polymorphisms of four bone mineral density candidate genes in Chinese populations and comparison with populations of different ethnicity. J Bone Miner Metab 2003; 21(1): 34-42.

39. Lau EMC, Choy DTK, Li M, Woo J, Chung T, Sham A. The relationship between COL1A1 
polymorphisms (Sp1) and COL1A2 polymorphisms (Eco R1 and PvuII) with bone mineral density in Chinese men and women. Calcif Tissue Int 2004; 75(2): 133-137.

40. Garnero P, Borel O, Grant SFA, Ralston $\mathrm{SH}$, Delmas PD. Collagen $1 \alpha 1 \mathrm{Sp} 1$ polymorphism, bone mass, and bone turnover in healthy French premenopausal women: the OFELY study. J Bone Miner Metab 1998; 13(5): 813-817.

41. Qureshi AM, McGuigan FEA, Seymour DG, Hutchison JD, Reid DM, Ralston SH. Association between COL1A1 Sp1 alleles and femoral neck geometry. Calcif Tissue Int 2001; 69(2): 67-72.

42. Liu PY, Lu Y, Long JR, Xu FH, Shen H, Recker RR, Deng HW. Common variants at the PCOL2 and Sp1 binding sites of the COL1A1 gene and their interactive effect influence bone mineral density in Caucasians. J Med Genet 2004; 41(10): 752-757.

43. Garcia-Giralt N, Enjuanes A, Bustamante M, Mellibovsky L, Nogues X, Carreras R, DiezPerez D, Grinberg D, Balcells S. In vitro functional assays and haplotypes of two COL1A1-promoter SNPs. Bone 2005; 36(5): 902-908.

44. Wilcken B, Bamforth F, Li Z, Zhu H, Ritvanen A, Renlund M, Stoll C, Alembik Y, Dott B, Czeizel AE, Gelman-Kohan Z, Scararo G, Bianca S, Ettore G, Tenconi R, Bellato S, Scala I, Mutchinick OM, Lopez MA, de Walle H, Hofstra R, Joutchenko L, Kavteladze L, Bermejo E, MartinezFrias ML, Gallagher M, Erickson JD, Vollset SE, Mastroiacovo P, Andria G, Botto LD. Geographical and ethnic variation of the $677 \mathrm{C} \rightarrow \mathrm{T}$ allele of 5,10 methylenetetrahydrofolate reductase (MTHFR): findings from over 7000 newborns from 16 areas world wide. J Med Genet 2003; 40(8): 619-625.

45. Castro R, Rivera I, Ravasco P, Jakobs C, Blom HJ, Camilo ME, Almeida IT. 5,10 Methylenetetrahydrofolate reductase $677 \mathrm{C} \rightarrow \mathrm{T}$ and $1298 \mathrm{~A} \rightarrow \mathrm{C}$ mutations are genetic determinants of elevated homocysteine. Q J Med 2003; 96(4): 297303.

46. Rosenberg N, Murata M, Ikeda Y, OpareSem O, Zivelin A, Geffen E, Selgsohn U. The frequent 5,10-methylenetetrahydrofolate reductase C677T polymorphism is associated with a common haplotype in whites, Japanese and Africans. Am J Hum Genet 2002; 70(3): 758-762.

47. Golbahar J, Hamidi A, Aminzadeh MA, OmraniGR.Association of plasma folate, plasmatotal homocysteine, but not methylenetetrahydrofolate reductase C677T polymorphism, with bone mineral density in postmenopausal Iranian women: a crosssectional study. Bone 2004; 35(3): 760-765.

48. Abrahamsen B, Medsen JS, Tofteng CL, Stilgren L, Bladbjerg EM, Kristensen SR, Brixen K, Mosekilde L. Are effects of MTHFR (C677T) genotype on BMD confined to women with low folate and riboflavin intake? Analysis of food records from the Danish osteoporosis prevention study. Bone 2005; 36(3): 577-583.

49. Friso S, Choi SW, Girelli D, Mason JB, Dolnikowski GG, Bagley PJ, Olivieri O, Jacques PF, Rosenberg IH, Carrocher R, Selhub J. A common mutation in the 5,10-methylenetetrahydrofolate reductase gene affects genomic DNA methylation through an interaction with folate status. Proc Natl Acad Sci USA 2002; 99(8): 5606-5611. 